\title{
Gradhiva
}

GRADHI

Revue d'anthropologie et d'histoire des arts

$10 \mid 2009$

Présence africaine

\section{Recettes des dieux : esthétique du fétiche}

Paris, musée du quai Branly, et Arles, Actes Sud, 2009

\section{Thierry Bonnot}

\section{OpenEdition}

Journals

Édition électronique

URL : http://journals.openedition.org/gradhiva/1598

DOI : 10.4000/gradhiva.1598

ISSN : 1760-849X

Éditeur

Musée du quai Branly Jacques Chirac

\section{Édition imprimée}

Date de publication : 4 novembre 2009

Pagination : 220-223

ISBN : 978-2-35744-012-8

ISSN : 0764-8928

Référence électronique

Thierry Bonnot, « Recettes des dieux : esthétique du fétiche », Gradhiva [En ligne], 10 | 2009, mis en ligne le 03 décembre 2010, consulté le 21 septembre 2020. URL : http://journals.openedition.org/ gradhiva/1598; DOI : https://doi.org/10.4000/gradhiva.1598

Ce document a été généré automatiquement le 21 septembre 2020.

(c) musée du quai Branly 


\section{Recettes des dieux : esthétique du fétiche}

Paris, musée du quai Branly, et Arles, Actes Sud, 2009

Thierry Bonnot

\section{RÉFÉRENCE}

Recettes des dieux : esthétique du fétiche, Cat. exp. [Paris, musée du quai Branly, 3 février-10 mai 2009] par Nanette Jacomijn Snoep, Jean-Paul Colleyn, Philippe Beaujard et al., Paris, musée du quai Branly, et Arles, Actes Sud, 2009

1 Face à certaines entités, nous demeurons perplexes : s'agit-il d'une chose naturelle ou d'un artefact, du produit d'une fabrication humaine ou du résultat d'un processus non intentionnel d'érosion, d'agglomération, de fusion dû au vent, à la pluie ou aux variations de températures? À quoi cela a-t-il bien pu servir? Que signifie cet assemblage ? Bref, qu'est-ce que ce «truc» ou ce "machin», comment le nommer, dans quelle catégorie l'inscrire? C'est à cette perplexité que nous confrontait l'étonnante exposition "Recettes des dieux", qui s'est tenue du 3 février au 10 mai 2009 au musée du quai Branly. L'objectif était précisément de montrer au public des éléments de collections rarement extraits des réserves, des choses invisibles et pourtant omniprésentes car intimement liées à la vie sociale de certaines populations ; peut-être d'ailleurs, sous une forme ou sous une autre, de toute population humaine. 


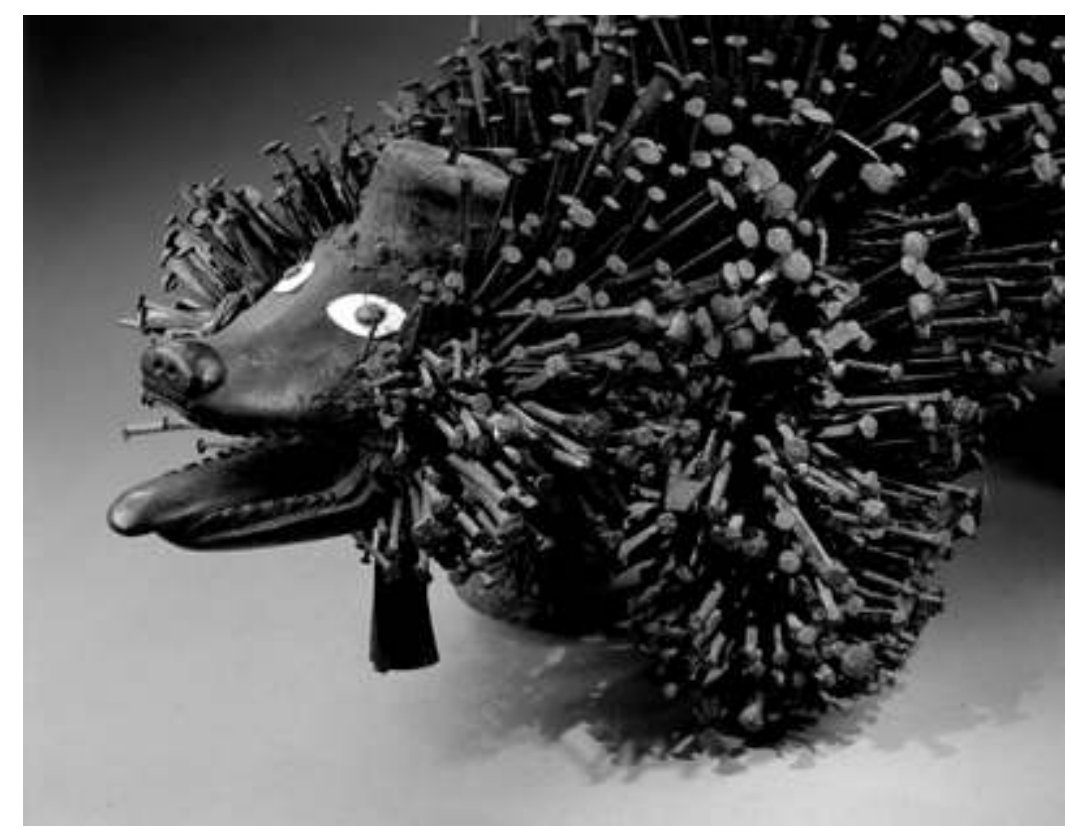

2 D'emblée, il est flagrant qu'il s'agit là d'objets inhabituels pour les visiteurs de ce musée. Ils ne correspondent pas aux canons esthétiques ordinaires des amateurs d'« art premier ", contrairement par exemple aux statues anthropomorphes du Pacifique de l'exposition «Mangaréva », présentée au même moment sur la galerie suspendue. À la perfection formelle de ces dernières, fruit d'un savant travail de taille et de polissage du bois, s'oppose l'indétermination des amas de crins, de tissu, d'ossements, de ferraille, de sang coagulé et de coquillages qui caractérisent ces objets de divination, des boliw, des emblèmes, des amulettes, des nkisi nkondi, des mohara de Madagascar ou de simples nœuds de liane rassemblés dans « Recettes des dieux ». Le pari, audacieux, consiste donc à présenter une exposition-dossier qui soit un «hommage aux choses informes », pour reprendre les termes de la commissaire d'exposition Jacomijn Snoep dans le remarquable petit catalogue édité pour l'occasion. Pour un musée que l'on a souvent accusé d'esthétisme - sous-entendu, antiscientifique-, c'est l'occasion de mettre en valeur des choses innommables, ignobles dans leur composition - croûtes, poils, sang séché, ossements... -, ne relevant pour la plupart d'aucun investissement ni activation esthétiques ${ }^{1}$, malgré le sous-titre donné à l'exposition. Il faut voir dans celuici, «Esthétique du fétiche ", une concession bien comprise à l'affichage promotionnel, le terme "fétiche» n'étant jamais utilisé dans les cartels, qui privilégient les désignations vernaculaires. Toujours dans le registre de l'esthétisation incongrue, on regrettera au passage le parti pris obscur de la muséographie : pourquoi faut-il qu'on soit obligé de s'arracher les yeux à tenter de déchiffrer les textes, comme s'il était préférable que le visiteur ne les lise pas, pour mieux le cantonner à la pure jouissance de la chose ? Ce parti pris semi-nocturne, assumé dès l'origine par les responsables du musée pour la présentation permanente du plateau des collections et maintes fois dénoncé, montre encore ici ses limites. Cet « éclairage spectral », obligeant « le visiteur à faire inéluctablement l'expérience des ténèbres» (Vogel 2007: 178), veut nous renvoyer à une Afrique magique, à son mystère, à ses nuits sauvages, en contradiction totale avec l'ambition affichée de la commissaire d'exposition, réfutant cette approche et dont tout le travail prouve effectivement son souci de clarification.

3 Nous voici face à l'innommable, l'informe, l'évolutif, l'inachevé. Ces réceptacles, objetspoèmes, objets-discours mais aussi objets-performances, résistent à nos catégories les 
plus familières. Ils mettent en cause nos certitudes, tellement inébranlables dès que nous observons le monde matériel, armés de nos convictions archéologiques faites de données concrètes et vérifiables - matières, techniques de fabrication, fonctions, datations, etc. Jean Bazin, ethnologue cité à plusieurs reprises dans l'exposition, avait prévenu contre cette assurance présomptueuse au sujet d'un nkisi nkondi qu'il confronta à La Joconde : «Le commentaire que nous fournit le musée, dans la mesure où il se veut simplement descriptif, devrait plutôt comporter une mention du genre composition en bois avec clous et autres matériaux. Dire que c'est un fétiche oriente vers un certain usage - la magie des primitifs, les croyances ("irrationnelles") des "autres"... On en dit déjà trop, plus que ne l'exige le simple "respect" de la chose" (Bazin 2008: 531). Chacun croit savoir, empiriquement ou savamment, ce que sont une statuette, un masque, un costume rituels. Mais où classer l'assemblage d'une mâchoire humaine et d'un crâne d'iguane séché, un ensemble de crins de cheval agglutiné en son sommet en une boule noirâtre de sang, de terre et d'excréments coagulés? Que faire de ces plumes, de ces chaînes métalliques, de ces morceaux de toile grossière, de ces pattes de volaille, de ces coquillages et cailloux sans valeur? Et encore ne voit-on ici qu'en photographies les amas gluants ou les simples traces dans le sable jouant également un rôle divinatoire... Ce sont des choses extra-ordinaires, littéralement; pas au sens où elles seraient réservées à des situations hors du commun, car certaines d'entre elles furent utilisées ou simplement portées quotidiennement, mais parce qu'elles échappent à la plupart de nos classifications et ne se plient que difficilement à nos habituels fichiers d'inventaire. La question est bien celle du classement légitime des objets et de leur détermination par l'usage. Fétiche et œuvre d'art ne sont pas immuablement stabilisés dans un usage religieux exotique ou un usage esthétique occidental : s'affranchir d'un tel fixisme permet de mieux comprendre ce que font les autres - ce que fait l'Autre.

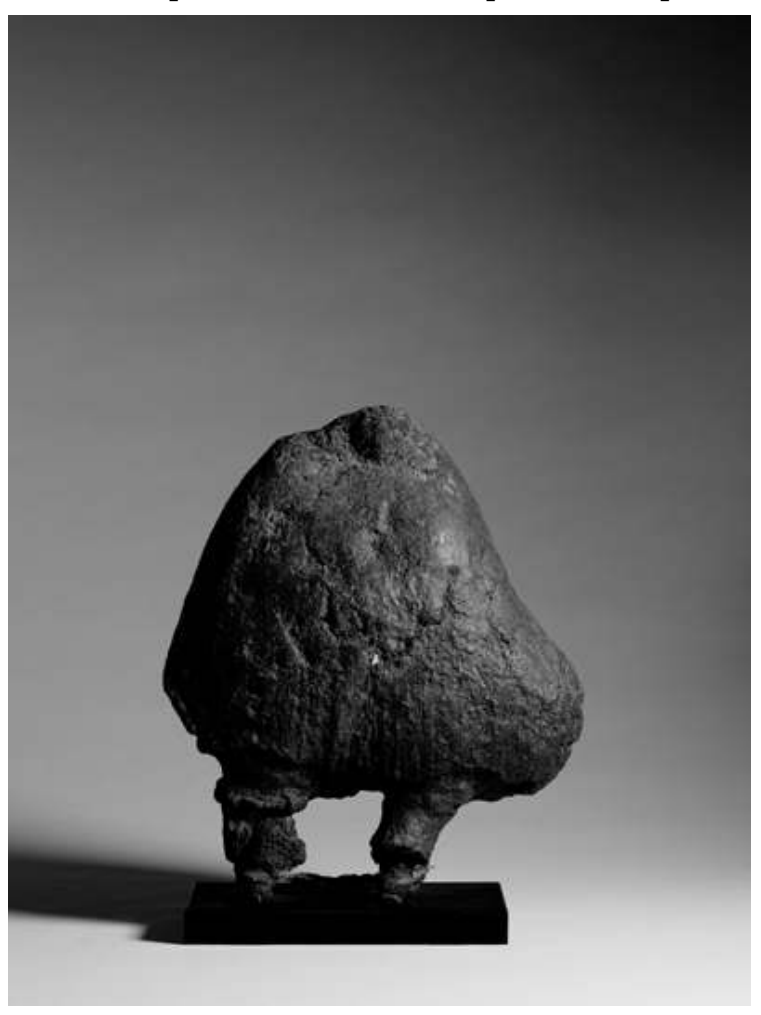

4 L'exposition "Recettes des dieux" assume pleinement ce parti pris. Les objets présentés obligent l'ethnologue à la modestie et à la rigueur descriptive plutôt qu'à 
l'exégèse interprétative. Faute de pouvoir attribuer a priori à ces choses des références statiques - en traduisant par exemple, de façon erronée et systématique, boli par autel (ibid. : 495) -, le chercheur doit apprendre sur le terrain ce qu'en font les hommes et ce qu'ils en disent. Mais ce sont là deux plans bien distincts que les responsables de l'exposition ont su ne pas confondre, évitant l'écueil de la surinterprétation. Car ce ne sont pas ici des significations qui sont assignées aux objets, mais des actions qui leur sont associées, inscrites dans un contexte local et un enchaînement historique. Prohibés par le pouvoir colonial et sa religion dominante, certains objets de divination devinrent objets de résistance et durent adopter une forme moins spectaculaire pour pouvoir mieux se dissimuler. D'autres compositions évoquent des variations de statut dépassant largement la question des croyances exotiques. Ainsi cet objet de divination bo du Bénin, une tête d'iguane attachée à une mâchoire humaine, trouvé sur le cadavre d'une amazone, guerrière de l'armée de Béhanzin lors de la bataille de Cotonou en 1890. Fétiche ou prise de guerre? Témoin d'une superstition de l'Autre ou d'une conquête coloniale ? Parfois, l'imaginaire spirituel et superstitieux peut être - littéralement !rattrapé par la réalité prosaïque, en l'occurrence par la justice coloniale : il en fut ainsi de ces amulettes qui servirent de pièces à conviction lors du procès d'une bande de voleurs togolais dans les années 1930. Chargés de leur conférer la force et la protection de quelque divinité, ces assemblages devinrent des preuves de leur culpabilité. En somme, ici, des objets témoins à charge. Histoire politique et connaissance ethnologique sont décidément inextricables et il est toujours salutaire de le rappeler, comme il est bon de remettre sur le métier les notions d'identité et d'authenticité : l'incorporation d'objets importés d'Europe aux agglomérats divinatoires nous interpelle sur leur appartenance définitive à un territoire, à une religion ou à une ethnie. Telle cette tunique protectrice de chasseur, chose-dieu sur laquelle on a accroché des extraits du Coran, comme une superposition de croyances et de religions.

Davantage que le produit final, l'objet qui nous est ici et maintenant donné à voir, c'est le processus qui importe: d'où l'organisation de l'exposition en une succession d'actions, envelopper, enduire, rembourrer, clouer, accumuler, etc.; autant de pratiques dont les objets sont à la fois le résultat et la condition d'efficacité. Par leur facture, par leur ordonnancement et le discours que traduisent les cartels, le visiteur est amené à voir les actions rituelles, sans qu'il soit besoin de les montrer, hormis par un extrait de film en fin de parcours, qui relate une séance d'initiation ${ }^{2}$. Ces compositions qu'on nous donne à voir sont à la fois des artefacts et des choses naturelles, des produits manufacturés et des ossements, des sculptures et des plumes de poulet, qui sont liés, articulés, saucissonnés, confondus par une même action rituelle au mépris de l'ontologie et des distinctions catégorielles ordinairement imposées à ses collections par le musée. Ils sont actions et gestes et ne représentent rien d'autre, comme l'affirmait Marc Augé, référence ethnographique sur le sujet: "L'exubérante matérialité des objets-fétiches, ces dieux de pleine terre, infirme par avance toute interprétation qui voudrait y voir la représentation d'autre chose : ils sont d'abord euxmêmes, pur amoncellement de substances organiques, minérales et végétales, agressivement matériels " (Augé 1988: 87). À aucun moment les textes de l'exposition ne prétendent donner l'ultime interprétation d'un fétiche et du rituel auquel il est associé : car sa recette est intimement tributaire des actions des hommes qui les manipulent et les constituent en même temps. Extraire l'objet de cette situation toujours singulière, c'est se couper de l'effet produit par l'interaction rassemblant lieux, hommes et femmes, objets, animaux, végétaux, soleil et pluie. Prétendre 
ressusciter cet effet derrière les vitrines d'un musée aurait été illusoire. La collecte, quelle qu'ait été sa nature - achat, vol, réquisition, don-, a interrompu un processus par lequel les objets se chargent de matières mais aussi de récits, de paroles et de gestes. Leur métamorphose s'arrête-t-elle pour autant? La mise en vitrine, nous dit Jacomijn Snoep, « est ainsi comme la fixation du dernier geste accompli sur l'objet ». Peut-être que, malgré la mise en réserve et l'attribution d'un numéro d'inventaire, certains agglomérats organiques continuent à travailler en sourdine, perpétuant ainsi la dynamique de la chose?

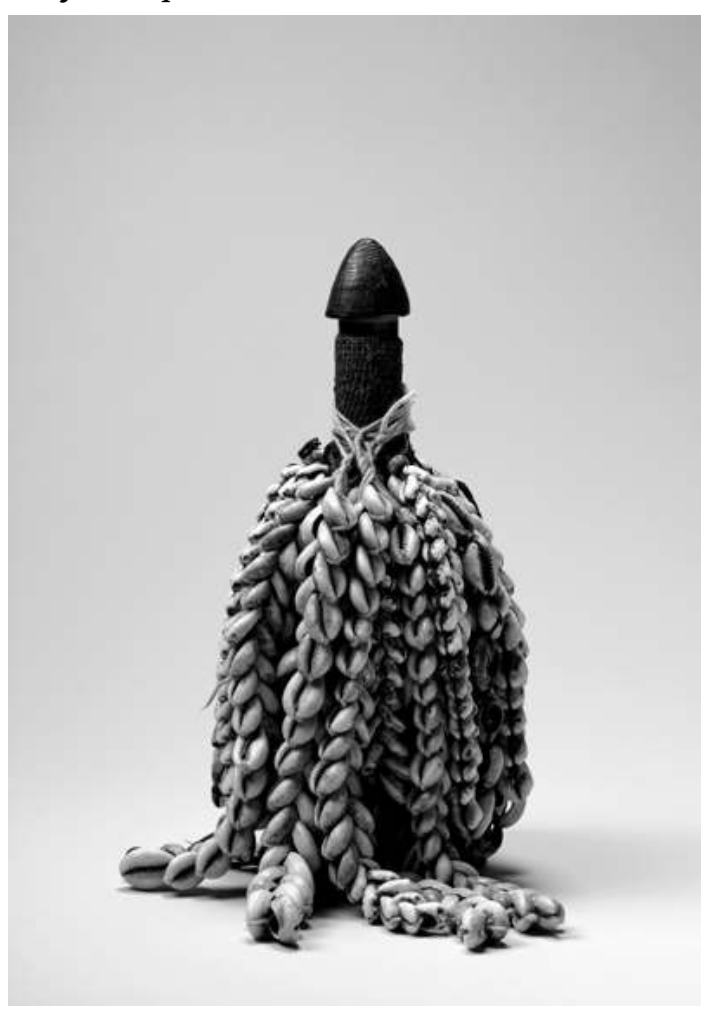

6 Pour conclure, il faut saluer l'excellente idée d'avoir présenté dans la dernière vitrine les objets déformés par l'éruption de la Montagne pelée en 1902, des verres principalement, repérés par André Breton qui en fit des «objets perturbés » exposés à la galerie Charles Ratton en 1936. Ce choix les transforma de fait en objets surréalistes, fétichisés par Breton comme sont fétichisés par un conservateur de musée un costume folklorique, une vieille paire de sabots, une charrue, une calebasse ou une coiffe de cérémonie. Immobilisés dans une vitrine, la continuité de leur devenir s'interrompt et les voici sacrés, fétiches du savoir ethnographique ou du culte patrimonial. N'importe quel objet peut devenir fétiche: nous sommes tous des féticheurs. "Recettes des dieux » proposait en somme une métaphore de l'objet fétichisé par le musée : comme les boliw, les objets entrant au musée ont accumulé de la force, des charges symboliques, des récits et ont changé de statut ou de nom au cours de leur parcours. L'acquisition, l'entrée en réserve, l'inventaire et le vernissage d'exposition peuvent être considérés comme autant de rites. C'est l'histoire de l'objet de musée que nous raconte le fétiche, mais aussi l'histoire de tout objet auquel s'attache un individu, ce qui suscite l'intérêt, le mystère et la sacralisation.

7 En rejetant tout positivisme monolithique par la thématique et le point de vue choisis, cette exposition stimulante constituait plus largement un raccourci problématique pertinent pour penser le rapport de l'ethnologie, voire des sciences sociales aux objets : 
que peuvent-ils signifier en dehors de l'action et surtout en dehors de l'interaction à laquelle ils participent? Pas plus que toute situation sociale, les objets matériels n'échappent au règne de l'incertitude dans laquelle se débat toujours le chercheur, notamment l'ethnologue. Il restera toujours un flou signifiant autour de ces trucs et de ces machins : mais cela n'est-il pas le lot de tous les objets en perpétuel devenir qui peuplent nos existences?

\section{NOTES}

Nkisi Nkondi, statuette zoomorphe magique, Congo (c) musée du quai Branly, photo Hughes Dubois. Boli. Pièce de bois recouverte de matériaux organiques et de sang animal coagulé. Fin xix-début xx siècle, Mali @ musée du quai Branly, photo Patrick Gries, donnation de Jean-Michel Huguenin.

Amulette ou « sika » liée aux jumeaux. Fabriquées par les jumeaux adultes pour les mères de jumeaux dont les enfants sont morts en bas âge, elle doit empêcher que leurs futurs enfants ne meurent jeunes. Bénin @ musée du quai Branly, photo Michel Urtado, Thierry Ollivier.

1. Pour reprendre les concepts de Jean-Marie Schaeffer, qui précise toutefois : «agir comme fétiche n'interdit pas de plaire esthétiquement » (Schaeffer $2004: 36$ ).

2. Julien Bonhomme, Voir l'invisible. Une initiation au Bwete Misoko (Gabon), film DV, 35 min, soustitrage français, 2003.

\section{AUTEURS}

\section{THIERRY BONNOT}

Thierry.bonnot@ehess.fr 